\title{
Systematic Search For Evidence of Tetrahedral and Octahedral Symmetries in Subatomic Physics: Follow-up of the First Identification Case in ${ }^{152} \mathrm{Sm}$
}

\author{
Jerzy Dudek ${ }^{1,2, *}$, Irene Dedes ${ }^{2}$, Abdelghafar Gaamouci ${ }^{3}$, Jie Yang $^{2}$, Andrzej Baran ${ }^{2}$, Dominique Curien ${ }^{1}$, Timo Dickel ${ }^{4,5}$, \\ and Hua-Lei Wang 6 \\ ${ }^{1}$ Université de Strasbourg and IPHC, 23, rue du Loess, F-67037 Strasbourg, France, CNRS, UMR7178, F-67037 Strasbourg, France \\ ${ }^{2}$ Instytut Fizyki - Uniwersytet Marii Curie-Skłodowskiej, pl. Marii Curie-Skłodowskiej 1, PL-20031 Lublin, Poland \\ ${ }^{3}$ Laboratory of Theoretical Physics, Faculty of Physics, USTHB, BP 32, El Alia, 16111 Bab Ezzouar, Algiers, Algeria \\ ${ }^{4}$ II. Physikalisches Institut, Justus-Liebig-Universität Gießen, 35392 Gießen, Germany \\ ${ }^{5}$ GSI Helmholtzzentrum für Schwerionenforschung GmbH, 64291 Darmstadt, Germany \\ ${ }^{6}$ School of Physics and Engineering, Zhengzhou University, Zhengzhou 450001, China
}

\begin{abstract}
In a recent article [1] group-theory representation-methods have been combined with the realistic mean-field calculation results to elaborate new, specifically designed methods of experimental identification of the tetrahedral/octahedral symmetries in atomic nuclei. The authors demonstrated that experimental data on ${ }^{152} \mathrm{Sm}$ existing in the literature are fully compatible with the extremely restrictive group-theory criteria of simultaneous presence of tetrahedral and octahedral symmetries, thus identifying these symmetries in subatomic physics for the first time. We discuss theory predictions related to the systematic presence of these symmetries as well as their manifestations throughout the Periodic Table in the form of islands centred around the doublymagic tetrahedral-symmetry nuclei.

The corresponding theory predictions are discussed in the context of the planned new experiments, which would employ the advanced mass-spectrometry methods [2], in view of the new experimental search criteria [1]. The addressed field of symmetry-research presents particularly promising potentialities in the domain of exotic nuclei studies. Indeed, as it can be demonstrated, in the exact tetrahedral and/or octahedral symmetry limits the corresponding nuclei emit neither E2 nor E1 radiation generating isomeric states with lifetimes which can become much longer than those of the related ground states. This is expected to open the new research strategies for the whole domain of the exotic nuclei studies throughout the Periodic Table.
\end{abstract}

Keywords: Nuclear structure, Nuclear Platonic symmetries, Exotic nuclei, Shape isomers

\section{Introduction: Mechanisms Associated with High-Rank Symmetries}

The issue of identifying exotic tetrahedral and octahedral symmetries among possible symmetries of nuclear shapes received increasing attention in recent years, cf. e.g. [3-9] and references therein. The corresponding groups of symmetry are usually denoted in the literature $\mathrm{T}_{d}$ and $\mathrm{O}_{h}$ and sometimes referred to as Platonic or in the nuclear physics context as high-rank. The origins of this interest are numerous and involve fascinating, often unprecedented exotic quantum mechanisms. Due to size limitations, this article presents only a brief description of mechanisms and phenomena whose observation will facilitate finding the evidence of high-rank symmetries in subatomic physics.

\subsection{Exotic Degeneracies of Energy Levels}

Among the most exotic unprecedented quantum features related to high-rank symmetries in nuclei let us mention the presence of characteristic four-fold degeneracies of the

*e-mail: Jerzy.Dudek@iphc.cnrs.fr single nucleonic energy-levels. They are predicted to occur in energy spectra generated by the mean-field Hamiltonians invariant under the high-rank symmetry groups [10].

The four-fold degeneracies originate from the characteristic structures of irreducible representations of the two groups. More precisely, the groups $\mathrm{T}_{d}$ and $\mathrm{O}_{h}$ just mentioned describing the forms of geometrical shapes, e.g. molecular ones in a 3D space and/or the tetrahedral and octahedral symmetry properties of the Hamiltonians of systems composed of bosons, have the well known partnership relations with the so-called double point-groups denoted $\mathrm{T}_{d}^{D}$ and $\mathrm{O}_{h}^{D}$, the latter describing the symmetries of the Hamiltonians of the systems composed of Fermions. Whereas $\mathrm{T}_{d}^{D}$ has two 2-dimensional and one 4-dimensional irreducible representations, the $\mathrm{O}_{h}^{D}$ has four 2-dimensional and two 4-dimensional irreducible representations. The wave-functions of the states transforming according to the 4-dimensional irreducible representations form quadruplets resulting in the 4-fold degenerate nucleonic energy levels.

It follows that in the exact symmetry limits those levels may lead to 16-fold and 32-fold degeneracies of the 
particle-hole excited states. Identification of these states in nature together with the identification of the underlying 4-fold degeneracy nucleonic orbitals remains today one of the challenges in the discussed domain of research.

\subsection{Unprecedented Structures of Rotational Bands}

Another exotic and at the same time fascinating issue related to the structure of tetrahedral-symmetry nuclei are the rotational bands with the totally new, unprecedented properties. Indeed, in contrast to the rotational bands studied traditionally, the latter composed exclusively of the $\Delta I=2 \hbar$ transitions connecting states with either positive or negative parities and composed of either even or odd spin states, the new rotational structures (let us focus on even-even nuclei to start with) are composed of certain even and certain odd spin states, the sequences including both parities and in addition containing degenerate multiplets. For instance, after $[11,12]$ and references therein, a rotational band built on the tetrahedral symmetry $I^{\pi}=0^{+}$ ground-state forms the spin-parity sequence ${ }^{1}$ :

$$
\underbrace{I^{\pi}=0^{+}, 3^{-}, 4^{+}, \underbrace{\left(6^{+}, 6^{-}\right)}_{\text {doublet }}, 7^{-}, 8^{+}, \underbrace{\left(9^{+}, 9^{-}\right)}_{\text {doublet }}, \underbrace{\left(10^{+}, 10^{-}\right)}_{\text {doublet }}, 11^{-}, \cdots}_{\text {Forming a common parabola: Irrep. } \mathbf{A}_{\mathbf{1}}}
$$

The above structure in the literature referred to as $A_{1}$, can be seen as one example among in total 5 irreducible representations of the $\mathrm{T}_{d}$-group describing tetrahedral symmetry properties of integer-spin rotational-states of even-even nuclei. The remaining characteristic rotor structures associated with the remaining irreducible representations $A_{2}$, $E, F_{1}$ and $F_{2}$ of the $\mathrm{T}_{d}$-group have equally exotic structural features, cf. [10, 11, 13] and references therein. For odd-even nuclei the characteristic spin-parity sequences are composed of half-integer spins. Identifying experimentally such very unique structures predicted by group theory can be seen as the strongest argument in favour of the final identification of the symmetries themselves. The first case of such an identification in ${ }^{152} \mathrm{Sm}$ can be found in [1].

\subsection{Abundance of Shape Isomers: Isomeric Bands}

Another highly exotic feature in the context of the collective rotation of tetrahedral/octahedral-symmetric nuclei is related to the fact that at the exact symmetry limit the electric quadrupole and dipole transition probabilities vanish, $B(E 2)=0$ and $B(E 1)=0$, [4]. It follows that the lowest multipolarity of non-vanishing electric transitions is $\lambda=3$ (i.e. the octupole transitions) which are orders of magnitude slower as compared to the $E 2$ (and/or E1) transitions. Recall that the quadrupole transitions dominate in the decay schemes of the "traditional" quadrupoledeformed collectively rotating nuclei and it follows that the states listed in Eq. (1) or analogous ones generated by the irreducible representations (irreps.) $A_{2}, E, F_{1}$ and $F_{2}$, are expected to become in fact long-lived isomers

\footnotetext{
${ }^{1}$ Spin-parity projected Hartree-Fock calculations demonstrate that the increasing size of the corresponding deformations increases the precision with which the discussed sequences form parabolic $E$-vs.- $I$ relations.
}

whose ensembles placed on the $(E-v s .-I)$-plane form parabolas, [13] and references therein. The condition that isomers form a specific smooth-looking relations of the type $E_{\text {isomer }}(I) \propto I^{2}$ is unprecedented in the nuclear structure physics and represents a possible focus for the newly established collaborations, cf. discussion accompanying Fig. 1 below.

\subsection{Symmetry-Breaking By the Zero-Point Motion}

Even though vanishing of the $B(E 2)$ and/or $B(E 1)$ reduced transition probabilities is a consequence of the exact tetrahedral/octahedral symmetry limits, such conditions are met strictly speaking only at the minimum-points on the potential energy surfaces corresponding to vanishing quadrupole (and other than tetrahedral/octahedral) deformations. However, according to the elementary properties resulting from the collective model of Bohr, deformations are not static and their probability distribution are described by the corresponding collective wave functions.

In particular for the lowest-energy single-minimum states these functions resemble Gaussians and the associated most probable (in particular quadrupole) deformations are not vanishing. This implies that there exist non-vanishing, even if small, associated "residual" reduced $B(E 2)$ or $B(E 1)$ electromagnetic transition probabilities connecting some tetrahedral symmetry states among themselves or with some other states within the spectrum. These probabilities can be estimated theoretically and can be used to identify traces of their presence with the help of the big multi-detector systems such as AGATA in Europe or GRETA in the USA. The corresponding experimental studies represent a powerful technique of investigations in line with the first tetrahedral/octahedral-symmetry identification-article addressing ${ }^{152} \mathrm{Sm}$, c.f. [1].

\subsection{Symmetry Breaking By Polarisation Effects}

Predictions of the high-rank symmetric nuclear configurations are based on the microscopic total energy calculations which suggest a presence of the well pronounced total energy minima corresponding to those exotic shapes which are surrounded by significant potential barriers. In the mean-field calculations such minima originate from the strong single-nucleon energy gaps giving rise to 'tetrahedral magic numbers' - analogues of the well known spherical magic gaps and spherical magic numbers.

However, when adding some nucleons on top of the magic-gap configurations (alternatively subtracting some) we perturb the symmetry similarly to the polarisation of the spherical configurations by extra particle or extra hole configurations. In other words the presence of the extra particles or holes polarises high-rank symmetric doublymagic cores leading to a partial symmetry breaking.

Similar partial symmetry breaking is expected as the result of the Coriolis polarisation effects induced by an increasing collective rotation. Indeed, the presence of collective angular momentum distinguishes an extra direction 
is space, the mechanism which breaks the original symmetry induced by the $\mathrm{T}_{d}$ and / or $\mathrm{O}_{h}$ symmetries and increases with increasing spin.

Perhaps paradoxically, both these symmetry breaking mechanisms can be helpful in identifying experimentally the discussed symmetries - under the condition that they are strong enough to induce small (residual) $B(E 2)$ [or $B(E 1)]$ collective reduced transition probabilities but at the same time sufficiently weak so that they do not destroy the symmetries in question entirely.

\subsection{Symmetry Driving Single-Nucleon Orbitals}

It turns out that the presence of strong gaps in the single nucleon spectra at high-rank symmetry shapes induces the presence of specific orbitals with particularly strongly down-sloping (usually above the gap) or up-sloping (usually below the gap) orbitals, cf. [14] for certain details. By an appropriate construction of the particle-hole excited states with the particle orbitals selected as down-sloping and hole states as up-sloping one may obtain configurations whose energies decrease by several (up to 10) $\mathrm{MeV}$ or so, leading to the final minima at the very significant high-rank symmetry deformations.

This mechanism allows for constructing the particlehole excited states with strongly increased tetrahedral / octahedral deformations. This may be particularly useful when studying the rotational properties of nuclei in the presence of these symmetries since the structures like the one in Eq. (1) are getting better and better pronounced (e.g. the doublet energies more and more precisely respected) the larger the symmetry-inducing deformation.

\subsection{Odd-A Nuclei as Tetrahedral Quantum Rotors}

Following semi-analytical results of [15], Coriolis coupling of an odd nucleon to the collective tetrahedral rotor takes a simple form involving mathematical expression with numerical constants referred to as generalised decoupling parameters. These expressions are more and more precisely respected by the full numerical result the stronger the symmetry-preserving deformation. They can be used to facilitate identifying the symmetry-induced mechanisms via comparison with experiment. It follows that employing the generalised decoupling parameters formulae provides a powerful symmetry identification method which is easy in applications.

\subsection{Symmetry-Oriented Research of Exotic Nuclei}

The states which in contrast to the "traditional" spectroscopic behaviour manifest neither $E 2$ nor $E 1$ collective transitions, the latter usually dominating the nuclear population and/or decay schemes are expected to form isomers thanks to the tetrahedral/octahedral-symmetry hindrance properties [13]. Their lifetimes, as compared to the life-times of many other states and in particular to the life-times of the ground-states, are expected to be significantly longer. Specifically in very exotic nuclei whose ground-state lifetimes are known to be extremely short their identification is at the limits of the instrumental capacities at present. Under these circumstances a possible existence of series of isomeric bands at low excitation energies opens potentially invaluable possibilities of populating states with sufficiently long lifetimes, possibly longer or much longer than the lifetimes of the ground-states. This would facilitate identifying the exotic nuclear species via long-living excited rather than short living groundstates. The use of the realistic theory-predictions will help optimising the experimental conditions in the specifically designed experiments to come, focussing on exotic and very exotic nuclei.

\subsection{New Search Strategies: Combining the Mass Spectrometry and $\gamma$-Ray Spectroscopy}

In the past, attempts have been undertaken to obtain an experimental evidence of tetrahedral/octahedral symmetries in nuclei by employing mainly the $\gamma$-ray spectroscopic methods. The contemporary Ge multi-detector arrays are becoming more and more powerful allowing to eliminate random coincidences and identify weaker and weaker transitions. However, the fact that nothing is known at present about the build-up of the symmetry breaking e.g. via zero-point motion, the extra particle-, or hole-states on top of the magic tetrahedral/octahedral configurations and/or Coriolis coupling in case of collective rotation increases even more the already significant difficulties in approaching the very weak feeding or decay out transitions engaging the high-rank symmetry states.

Under these conditions alternative approaches possibly leading directly to the information about the isomers without directly involving $\gamma$-spectroscopic information are extremely welcome. This type of information will be obtained by applying multiple-reflection time-of-flight mass spectrometry (MR-TOF-MS) with highest resolving power as developed at the Justus-Liebig-University, Gießen [2]. This approach will allow to measure isomeric states down to about $200 \mathrm{keV}$ of excitation energies [16]. Moreover, it will offer a possibility of measuring in a broadband mode so that many neighbouring nuclei which are predicted by theory to manifest high-rank symmetry configurations will be measured at the same time.

The nuclei of interest will be produced employing spontaneous fission process. We plan using ${ }^{252} \mathrm{Cf}$ spontaneous fission source installed in a gas-filled stoping cell. The MR-TOF-MS and the gas-cell are parts of the FRS Ion Catcher experiment at GSI, Darmstadt [17]. After thermalisation in the gas, the fission fragments will be extracted with the help of electric field and transported to the MR-TOF-MS. An illustration of the possible zones of research with this particular device compared with the distribution of the doubly-magic tetrahedral nuclei over the Periodic Table are illustrated in Figure 1. The combination of accelerator-independent, broad and stable production together with a very sensitive, universal and broadband detection methods allows long term studies of long time spans when measuring even the very weakly populated states. 


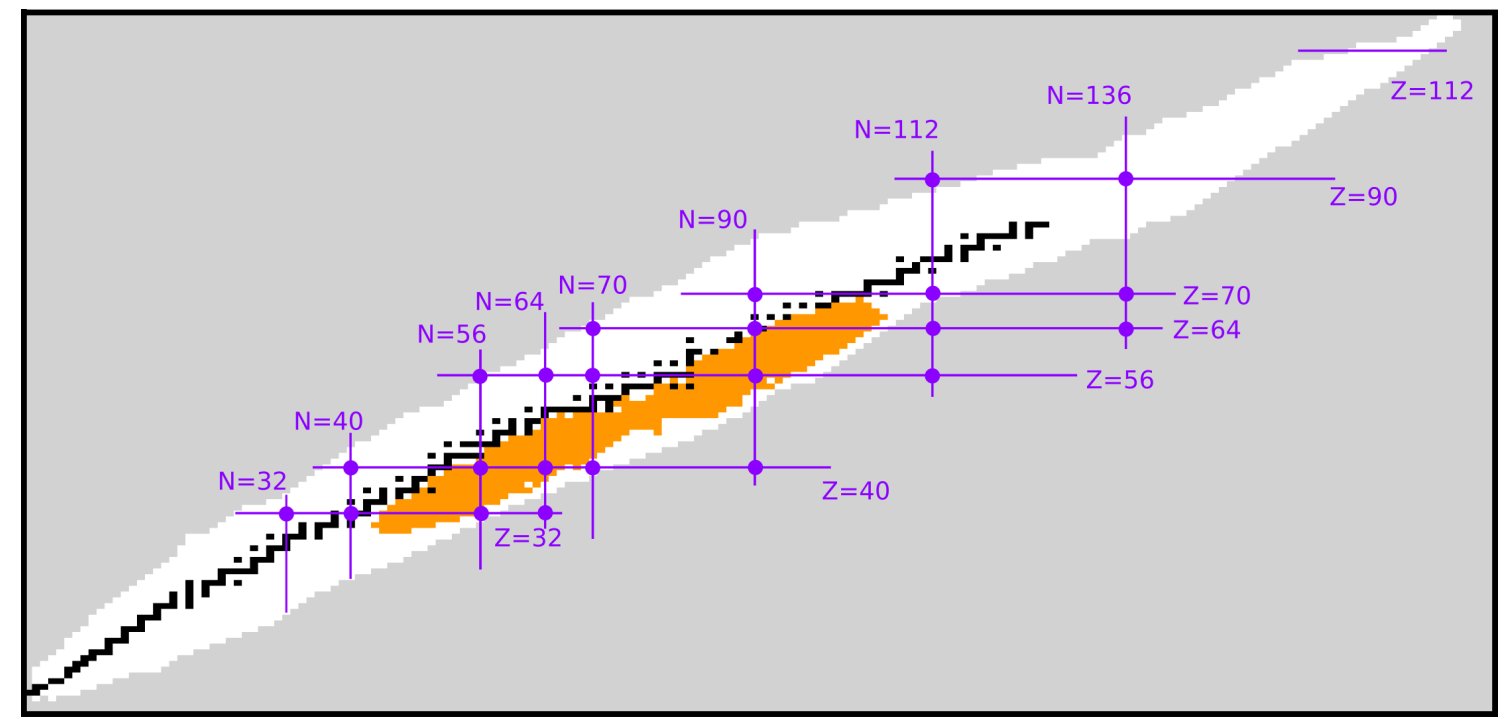

Figure 1. Positions - marked with the full circles - of the doubly-magic tetrahedral nuclei as predicted by theory. There is a relatively broad range of nuclei susceptible to produce the tetrahedral-symmetry configurations around each central doubly-magic tetrahedral nucleus as illustrated in figures in Section 2. The range of nuclei produced using ${ }^{256} \mathrm{Cf}$ source in the GSI set-up is marked explicitly.

\section{Viewing High-Rank Symmetries as Crypto-Symmetries: Illustrations}

At an early stage of theoretical investigations of the tetrahedral and octahedral symmetries in nuclei it became clear that the realistic nuclear mean-field calculations, both the phenomenological ones - based on the Woods-Saxon universal Hamiltonian [18], and the microscopic ones based on the Skyrme Hartree-Fock Bogolyubov Hamiltonians predict systematic occurrence of the high-rank symmetry configurations in numerous ranges of the Periodic Table. In other words, and in full accordance with the scheme presented in Figure 1, the nuclei for which the high-rank symmetry configuration are predicted are much more numerous then the nuclei with the well known spherical symmetry. And yet, until the very recent announcement [19] of the identification of the high-rank symmetries in a single nucleus ${ }^{152} \mathrm{Sm}$ there was no other case signalled. This situation inspired Dudek and coworkers [19] to introduce the nickname $\kappa \rho v \pi \tau o-\sigma v \mu \mu \epsilon \tau \rho \iota \epsilon$ (crypto-symmetries or hidden symmetries) which alludes to the fact that the high-rank symmetries, even though expected to be present allover, have never been seen experimentally - as we believe - mainly because so far nobody was looking for them with the designed methods, and thus the symmetries remain "hidden" (interested reader might compare with the techniques designed in [1]).

In the following we will present briefly certain predictions related to the scheme in Figure 1, in the context of the new approach proposed in Section 1.9. Let us begin by noticing that among 23 doubly-magic tetrahedral nuclei marked in the figure there are only five of them, viz. ${ }^{88} \mathrm{Ge},{ }^{96} \mathrm{Zr},{ }^{104} \mathrm{Zr}$, ${ }^{146} \mathrm{Ba}$ and, possibly, ${ }^{154} \mathrm{Gd}$, which are close to the zone of nuclei populated as fission fragments of ${ }^{252} \mathrm{Cf}$. All the five can be considered extremely exotic and therefore populated under very unfavourable conditions of statistics.
Consequently it will be of fundamental interest to learn what are the other, i.e. not necessarily doubly magic tetrahedral-symmetry nuclei predicted by theory which possibly present strong signatures of the tetrahedral or the octahedral symmetry configurations?

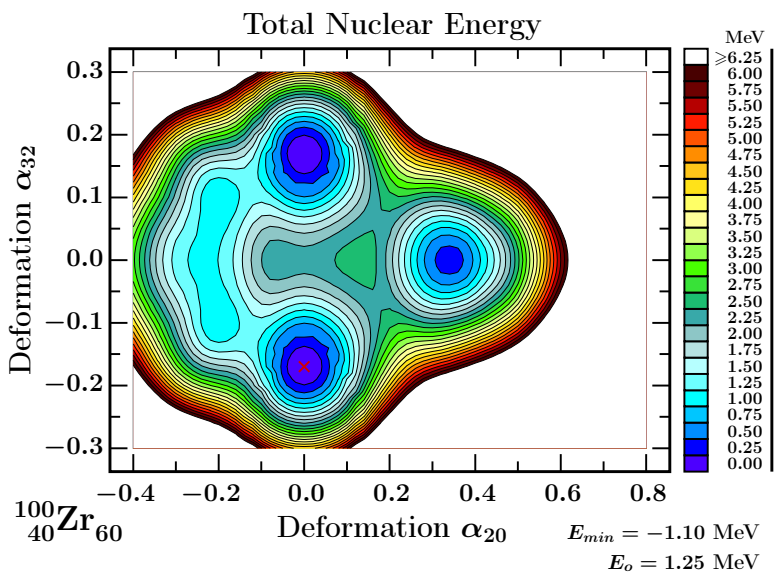

Figure 2. An example of the potential energy surface, here for ${ }^{100} \mathrm{Zr}$ nucleus on the plane spanned by the quadrupole $\left(\alpha_{20}\right)$ vs. tetrahedral $\left(\alpha_{32}\right)$ deformations using the phenomenological Woods-Saxon realisation of the mean-field approach with the macroscopic-microscopic method of Strutinsky. The symmetric tetrahedral minima at $\alpha_{32} \approx \pm 0.18$ are in direct competition with the strongly deformed axial-symmetry minimum at $\alpha_{20} \approx 0.37$. Similar illustrations apply for numerous nuclei within the fissionfragment population zone marked in orange colour in Figure 1.

To answer this type of questions we have performed systematic calculations using the realistic phenomenological mean-field approach with the deformed potential of the Woods-Saxon type using the so-called "universal" parametrisation of [18]. The Hamiltonian de- 
pends on 12 adjustable parameters whose values have been fixed once for all nuclei in the Periodic Table (wherefrom the name "universal" when referring to this parametrisation) using the experimental information about the singlenucleon levels in deformed nuclei. This parameterisation has been shown to describe numerous nuclear structure properties in a very satisfactory fashion, at least on average. Within our analysis, the total energy surfaces have been calculated in the space of deformations including $\alpha_{20}$ and $\alpha_{22}$ (quadrupole deformations), $\alpha_{32}$ (tetrahedral deformation) and $\alpha_{40}$ (hexadecapole deformation) using macroscopic-microscopic method of Strutinsky with the macroscopic energy defined after the so-called Yukawafolded approach.

Figure 3 shows a selection of the total energy results for Zirconium nuclei with $N \in[56,76]$ in the form of the one-dimensional cross-sections originating from the

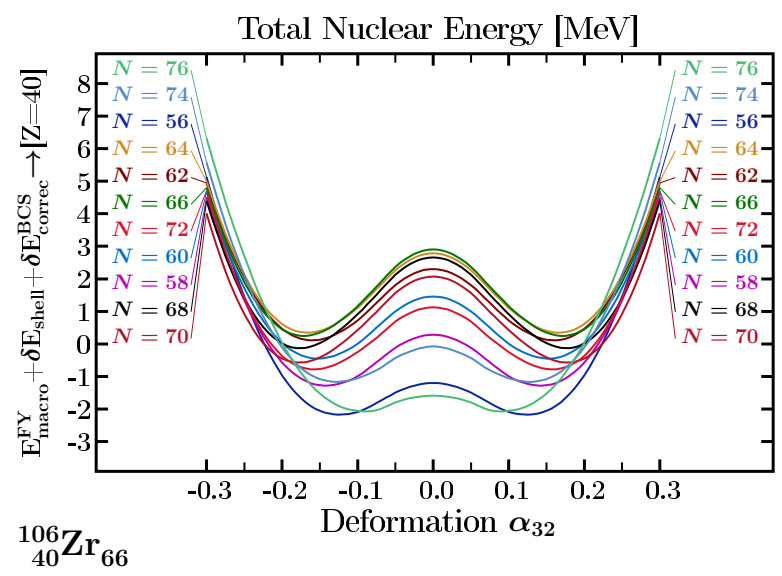

Figure 3. Cross-sections of the total energy surfaces like the one in Figure 2 but for a number of Zirconium isotopes showing the tetrahedral-symmetry minima not only for the magic numbers $N_{T_{d}}=56,64$ and 70 but also for numerous other isotopes. These theory predictions encourage experimental programme briefly announced in Section 1.9.

results like the ones in Figure 2. Let us notice that actually for all the nuclei displayed the total nuclear energies present tetrahedral-symmetry minima. Given the relatively high potential barriers separating the twin tetrahedral minima like the ones in Figure 2 from one another and comparably important barriers separating the two from the third ones, the large quadrupole-deformation minima, we conclude that according to the calculations there will be numerous nuclear states in the discussed mass range with the tetrahedral symmetry local minima expected to lead to longlived tetrahedral symmetry isomers.

Analogous results are illustrated in Figure 4 for a series of Gadolinium nuclei. From the results presented for Zirconium and Gadolinium isotopes shown but also from the similar comparisons for other elements we may conclude that the tetrahedral shell effects are very specific in the sense that they do not privilege strongly the tetrahedral doubly magic-number nuclei as compared to their neighbours. In reality, there are several dozens of various iso-

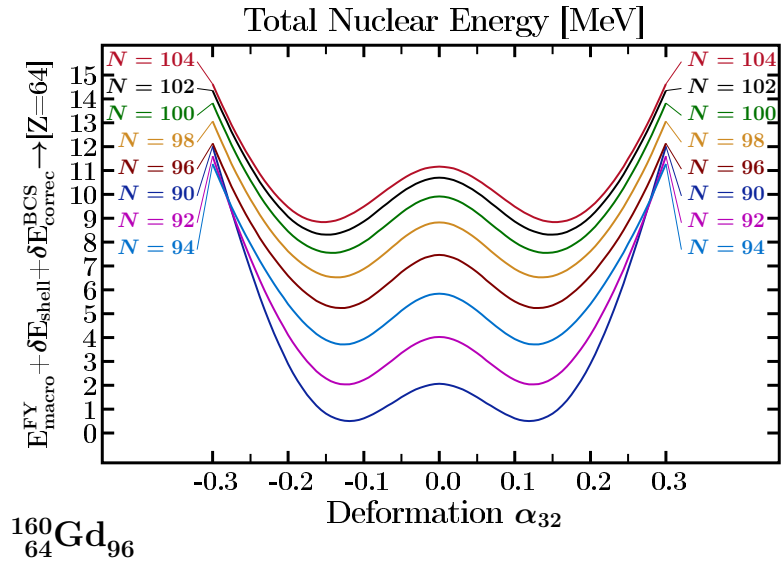

Figure 4. Cross-sections of the total energy surfaces like the ones in Figure 3 but for a number of Gadolinium isotopes showing the tetrahedral-symmetry minima not only for the magic numbers $N_{T_{d}}=90$ but also for numerous other isotopes. These theory predictions strongly encourage experimental programme briefly announced in Section 1.9.

topes and isotones which, according to the theory predictions should manifest tetrahedral symmetry configurations very likely leading to the isomers. Moreover, let us remind the reader that according to the arguments given in Sections 1.2 and 1.3 each tetrahedral symmetry minimum leads to numerous rotational states expected to lead to the isomers and/or bands composed of isomers.

\section{Summary and Conclusions}

In the first part of this article we recalled briefly the numerous exotic and most of the time unprecedented quantum effects expected to accompany the atomic nuclei in their tetrahedral and/or octahedral symmetry configurations. All these effects attracted certain attention in the literature in the past contributing to the growing interest in the exotic symmetries especially in the exotic nuclei. Whereas the quantum effects in question provide strong motivation for this kind of research per se, the existence of the hindrance factors which cause vanishing of the usually strong quadrupole and/or dipole electric transitions leads very likely to the novel class of shape isomers opening the new avenues for the studies of exotic nuclei for which ground-state life times are too short for the present day instrumentation.

Group theory considerations recalled briefly in this article lead to the new classes of rotational bands whose members can carry both parities and/or may form doublets, triplets, quadruplets, etc. All these features can be seen as unique experimental identification criteria of the states and phenomena involving these exotic symmetries. The expected existence of many isomeric states with tetrahedral/octahedral symmetries makes it natural to propose the mass spectrometry as one of the most efficient criteria of identification of such nuclear states. In this context we propose applying multiple-reflection time-of-flight 
mass spectrometry (MR-TOF-MS) in collaboration with the teams from the University of Gießen and from the GSI, Darmstadt in addressing the issue of identifying the presence of the isomers. Let us emphasise that theory predicts the presence of many high-rank symmetry (thus isomeric) states in the area of the Periodic Table covered by the ${ }^{252} \mathrm{CF}$ fission-fragment production zone in the GSI experiment foreseen soon.

Acknowledgements. This work was partially supported by the Polish National Science Centre under Contract No. 2016/21/B/ST2/01227 and Polish-French COPIN-IN2P3 collaboration agreement under project numbers 04-113 and 05-119.

\section{References}

[1] J. Dudek, D. Curien, I. Dedes, K. Mazurek, S. Tagami, Y. R. Shimizu, and T. Bhattacharjee; Phys. Rev. C 97, 021302(R) (2018)

[2] T. Dickel et al., Nucl. Instrum. Meth. A777 (2015) 172-188

[3] J. Dudek, A. Góźdź, N. Schunck and M. Miśkiewicz, Phys. Rev. Lett. 88, 252502 (2002)

[4] J. Dudek, D. Curien, N. Dubray, J. Dobaczewski, V. Pangon, P. Olbratowski, and N. Schunck, Phys. Rev. Lett. 97, 072501 (2006)

[5] J. Dudek, D. Curien, A. Góźdź, Y. R. Shimizu and S. Tagami, Acta Phys. Polonica B 44, 305 (2013)

[6] S. Takami, K. Yabana, and M. Matsuo, Phys. Lett. B 431242 (1998)

[7] M. Yamagami, K. Matsuyanagi, and M. Matsuo, Nucl. Phys. A 693579 (2001)

[8] K. Zberecki, P.-H. Heenen, and P. Magierski, Phys. Rev. C 79, 014319 (2009)

[9] J. Zhao, B.-N. Lu, E.-G. Zhao, and S.-G. Zhou, Phys. Rev. C 95, 014320 (2017)
[10] M. Hamermesh, Group Theory and Its Application to Physical Problems, Dover Publications, Inc., New York, 1962.

[11] S. Tagami, Y. R. Shimizu, and J. Dudek, Phys. Rev. C 87, 054306 (2013).

[12] S. Tagami, Y. R. Shimizu, and J. Dudek, J. Phys. G: Nucl. Part. Phys. 42, 015106 (2015).

[13] J. Dudek, I. Dedes, J. Yang, A. Baran, D. Curien, T. Dickel, A. Góźdź, D. Rouvel and H. L. Wang; Acta Phys. Polonica B 50, 685 (2019)

[14] A. Baran nad J. Dudek, Acta Phys. Polonica B Proceedings Supplement, 2019 in print

[15] S. Tagami, Y. R. Shimizu and J. Dudek, Phys. Rev. C 98, 024304 (2018)

[16] S. A. S. Andrés et al., Phys. Rev. C 99064313 (2019)

[17] W. R. Plaß et al., Nucl. Instrum. Meth. B 317457 (2013)

[18] The Universal Woods-Saxon Hamiltonian and associated, so-called 'universal parametrisation' has been developed in a series of articles:

J. Dudek and T. Werner, J. Phys. G: Nucl. Phys. 4, 1543 (1978),

J. Dudek, A. Majhofer, J. Skalski, T. Werner, S. Cwiok, and W. Nazarewicz, J. Phys. G: Nucl. Phys. 5, 1359 (1979),

J. Dudek, W. Nazarewicz, and T. Werner, Nucl. Phys. A 341 253, (1980),

J. Dudek, Z. Szymański, and T. Werner, Phys. Rev. C 23, 920 (1981), and has been summarised in

S. Ćwiok, J. Dudek, W. Nazarewicz, J. Skalski, and T. Werner, Comp. Phys. Comm. 46, 379 (1987).

This parametrisation is being used without modifications by many authors also today.

[19] J. Dudek, N. Schunck, N. Dubray and A. Góźdź, Int. J. Mod. Phys. E 14389 (2005) 